\title{
The Chemistry of Life
}

\author{
Boris Aleksandrovich Trofimov, laureate of the Russian Federation National Award \\ for substantial contribution to the development of new methods fine organic syn- \\ thesis in order to design innovative drugs and modern materials, including special- \\ purpose ones, tells us about his discoveries and further research plans.
}

- Boris Aleksandrovich, you are the author of over 500 inventions. Which one is the most significant to you?

- I would say I am a co-author. Very few inventors work independently now. It is a relic of a phenomenon. Today, researchers typically work in collaboration, so serious findings are a result of the joint efforts of experts from different fields. Practically significant ideas most frequently emerge at the fault lines of sciences. Therefore, most of our inventions have a cross-disciplinary character and are a result of the efforts of my students, collaborators, and colleagues from related areas of science and technology.

Which invention is the most significant to me? I think it is a rather difficult question, similar to the situation when the father of a large family cannot say who his favorite child is. In addition, attitudes to inventions have changed as time has passed. Not all the inventions made 20 or even 40 years ago still seem important today. On the contrary, some findings that used to seem quite ordinary have turned out to be uncut diamonds. Of course, our recent inventions are the most important ones for me, since they are relevant to the demands of modern life. Still, there are some inventions that I regard as key ones. They laid the groundwork for new directions of chemistry and chemical engineering and became so-called "idea incubators," yielding new inventions. These inventions include the methods for pyrrole synthesis from ke-

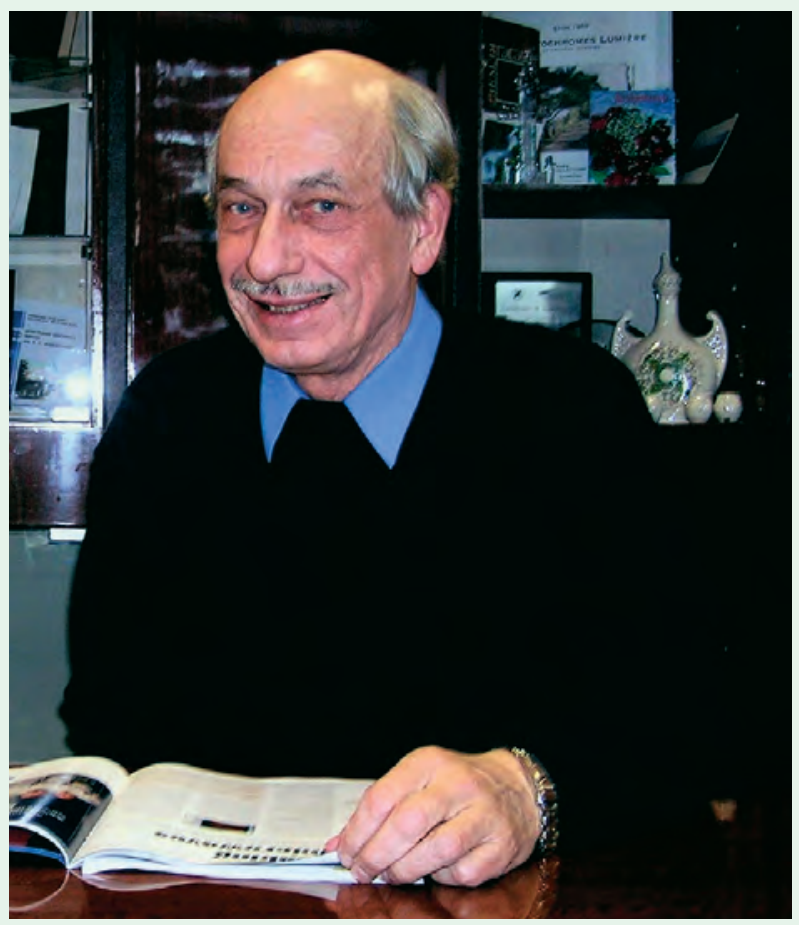

Boris Aleksandrovich Trofimov, Director of the A.E. Favorsky Irkutsk Institute of Chemistry, Siberian Branch, Russian Academy of Sciences, Doctor of Science (Chemistry), Professor, and Academician of RAS. Laureate of the A.M. Butlerov Award and the Russian Federation National Award.

tones and acetylene; direct chlorinefree synthesis of phosphororganic compounds from elementary phosphorus and acetylene or its derivatives; and the synthesis of divinyl chalcogenides (e.g., divinyl sulfides, divinyl selenides, divinyl tellurides) directly from elementary chalcogens or their simplest derivatives (such as hydrogen sulfide) using acetylene or its derivatives. I would also attribute the "anchor" epoxides (e.g., Vinylox) used to produce many new brands of epoxy resins with special techni- cal characteristics to the category of fundamental inventions.

- Pyrrole synthesis from ketones and acetylene is now known as the Trofimov reaction and has been included in a number of reference books, including the Encyclopedia of Chemical Technology (USA). Please tell us more about the reaction and what it is used for.

- Several approaches to pyrrole synthesis have been described so far; however, they either require expensive and complex starting 
compounds or involve several reactions (chemists refer to them as multistage approaches), or the pyrrole yield is low, or these synthesis methods are insufficiently universal (i.e., they yield only a limited number of pyrrole derivatives). Our reaction (see the scheme), which was discovered in the early 1970 s (jointly with junior researcher A.I. Mikhaleva (today, she is a professor)), requires only ketones, one of the most available and inexpensive classes of organic compounds, hydroxylamine, and acetylene, and even cheaper industrial products.

For example, the widely known acetone (the simplest ketone) reacts with hydroxylamine and acetone under mild heat treatment in the presence of a superbasic catalytic system, which consists of potassium hydroxide and dimethyl sulfoxide (used in medicine) and can be easily prepared, yielding $\alpha$-methylpyrrole and its N-vinyl derivative. By the way, until our reaction was discovered, N-vinylpyrroles remained an unknown, although very promising, class of pyrrole compounds.

Pyrrole and its derivatives are the fundamental structures of key life-sustaining systems; namely, chlorophyll, hemoglobin, natural antibiotics, insect pheromones, and a number of drugs. Our method for pyrrole synthesis is currently finding increasing application for producing pharmaceutical agents, biosensors, light-sensitive metal complexes for the diagnosis and therapy of cancer, molecular systems for artificial photosynthesis, and materials for new technologies (organic semiconductors, electro- and photochromic materials, and optoelectronic devic- es). The reaction discovered by us enabled to develop the world's first technology to produce synthetic indole from cyclohexanone and acetylene. Pilot plant production of indole with a capacity of 200 tons per year is currently being planned. Our technology enables the simultaneous production of tetrahydroindole (today, $1 \mathrm{~g}$ of this compound costs over 200 euro), its vinyl derivative, and $\mathrm{N}$-vinylindole, which are valuable intermediate products for the synthesis of pharmaceutical agents and are also unique monomers which are not produced anywhere in the worlds at the moment.

Indole is finding wide application in the synthesis of pharmaceutical agents and the important biologically active compounds regulating the vital activity of warm-blooded animals. Indole is the main structural element of such crucial compounds in nature as tryptophan (an essential amino acid), serotonin (one of the main neuromediators, the "happiness hormone"), and melatonin (the hormone regulating human circadian rhythms). Its structure can be found in other hormones and hallucinogens. Well known indole-based drugs include indopan, indometacin (anti-inflammatory, antipyretic, and analgesic agents), reserpine (a tranquilizer), and strychnine (a nervous system stimulant). Heteroauxin ( $\beta$-indolyl acetic acid), an indolebased plant growth stimulant, is widely used in practice.

- So why has acetylene drawn your attention?

- Indeed, we have designed and further developed the scientific principles of original methods for organic and element-organic synthesis

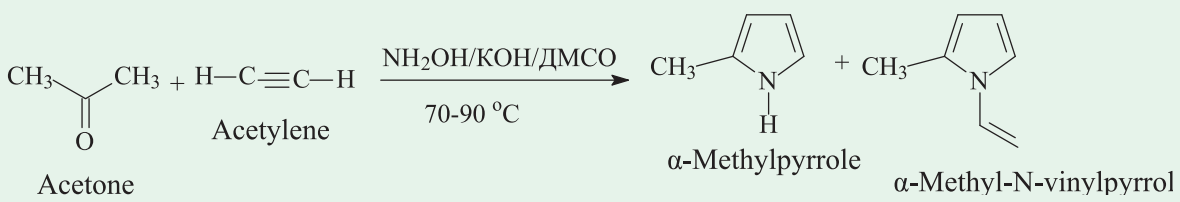

Scheme. Synthesis of $\alpha$-methylpyrrole and $\alpha$-methyl-N-vinylpyrrole from acetone and acetylene. based on acetylene. Acetylene is the simplest high-energy hydrocarbon, an obligate product of gas, coal, and oil processing, the fundamental unit of the organic matter. This gas has even been detected in interstellar space. It has been conventionally used and is still being used as a starting raw material for industrial and fine organic synthesis. In the XX century, it was used to synthesize all major chemical products. It has been replaced by ethylene and propylene produced from oil (which remain cheaper raw materials). However, it is now assumed that acetylene will return in the chemical industry in the nearest future due to the steady rise in the prices of oil and gas, since acetylene can also be produced from coal, material that will remain available for hundred of years. Acetylene is currently being increasingly used in fine organic synthesis oriented towards the production of complex science-intensive molecules (biologically active substances, vitamins, drugs, agricultural fertilizers, and high-tech materials) due to the fact that it enables to take the shortest and simplest pathway to the obtaining of the target compounds.

In the early 1960 s, when the Siberian Branch of the USSR Academy of Sciences was being established, Mikhail Fedorovich Shostakovsky, then a corresponding member of the USSR Academy of Sciences and favorite pupil of outstanding Russian organic chemist Aleksei Evgrafovich Favorsky, brought the research area and traditions of the school of his great teacher to the Irkutsk Institute of Organic Chemistry, Siberian Branch of the USSR Academy of Sciences. So a new and young branch of Academician Favorsky's school, whose scientific interests focused on acetylene chemistry and fine organic synthesis, was established and began to rapidly develop in Eastern Siberia. Most of the attention was always focused on the production and modification of biologically ac- 
tive substances; namely, terpenoids, steroids, alkaloids, and saccharides. Our institute was later named after Academician Favorsky for the successful development of the scientific heritage of this outstanding founder of acetylene chemistry. My pupils and I are proud to belong to his school.

- You have developed methods for fine organic synthesis of various chemical compounds, including pharmaceutical agents, dyes, chemical additives, pesticides, surfactants, and synthetic enzymes. Which pharmaceutical agents produced via fine organic synthesis are the most noteworthy?

- To be more precise, we have fundamentally supplemented the fine organic synthesis procedure. We have discovered a number of new reactions and have substantiated new methodological principles (e.g., the use of superbasic catalytic media, reactants, and catalysts). As a result, previously unknown ways for producing useful substances have emerged. Like other synthetic chemists, we search for new laws of formation of chemical bonds in order to perform a targeted and simple construction of high-tech materials, innovative compounds, and drugs, without which modern civilization cannot exist. It is not by mere chance that the recent Nobel Prize in chemistry was awarded for the methods of formation of the carbon-carbon bond via the substitution of atoms (typically, halogens) in the presence of noble metals. Our methods are based on another type of reactions, addition reactions, which are catalyzed by natural ions (sodium and potassium ions, hydroxide anion) or light quanta; therefore, these methods are usually wasteless. In other words, they satisfy the principles of green chemistry to a significant extent, are environmentally friendly and energy- and resource-saving (chemists refer to these principles as atom-economic methods).
All this is valid for the new type of reactions discovered and developed by my colleagues, academicians V.N. Charushin and O.N. Chupakhin, both laureates of the same National Award. Their reactions involve hydrogen replacement in the aromatic or heteroaromatic ring. The hydrogen being released does not pollute the environment and can be used as the "purest" fuel and chemical reactant. The new chemistry has allowed my friends and colleagues to complete the registration of the new antitumor agent lisomustin. This drug has been introduced into medical practice and is successfully used in clinics. They have developed new industrial methods for the synthesis of antibacterial fluoroquinolone antibiotics (perfloxacin and levofloxacin). Preclinical studies of the original antiviral drug triazavirin, which is capable of providing $90 \%$ protection against a number of dangerous viral infections (e.g., H5N1 and H1N1influenza), were recently completed.

Acizolum, a high-efficiency antidote of carbon monoxide, has been designed in our institute on the basis of $\mathrm{N}$-vinylimidazole obtained via direct vinylation of an essential heterocycle, imidazole, with acetylene. This antidote efficiently protects people against poisoning with this insidious gas and other combustion products. Acizolum has been included in Russia's Vital and Essential Drug List. This drug is produced by the MAKIZ Farma pharmaceutical company under the supervision of Acizol, a closed joint-stock company (Moscow). This drug is becoming more important as the number of fires increases all over the world. A number of additional valuable properties have recently been revealed for acizolum. In addition to being a unique antihypoxant, it is an active hepatoprotector (comparable to the best known drugs) and an antiarrhythmic agent. It allows one to cure the most severe pneumonias and even relieves hangover.
A high-efficiency antiseptic agent, anavidin, which was designed in our institute, is now produced by an especially established company on a setup with a capacity of 100 tons per year. The dynamically developing Russian pharmaceutical company Farmasintez in a few months intends to bring to market a new anti-tuberculosis agent, perchlozone, which is active against resistant mycobacterial species. This original drug took many years to design in our institute (starting from the first synthesis of the molecule in the laboratory headed by Professor G.G. Skvortsova) and, of course, is a product of fine organic synthesis. I would like to mention that no other anti-tuberculosis agents capable of efficient resistance to new aggressive strains of tuberculosis bacteria have appeared in pharmaceutical practice during the past decades.

- Boris Aleksandrovich, new environmentally friendly methods for synthesizing organic compounds of phosphorus and chalcogens have been developed in your institute...

- The synthesis of drugs (more frequently, drug precursors) and drug candidate molecules is significant in fundamental studies carried out in our institute. To be more precise, it is the applied stage of these studies. Our main purpose is to develop new methods for the synthesis of complex organic molecules and reveal the relationship between the structure of the synthesized compounds and their useful properties in order to increase efficiency and reduce the costs associated with the targeted synthesis of compounds that people currently need. The demand is not for drugs only. First of all, people need health. And it would be better if a person could be healthy without drugs. To achieve this, we need to reduce pollution of the environment, since a significant number of contemporary diseases are a result of our reckless and even crimi- 
nal attitude to nature. The methods for synthesizing phosphorus- and chalcogen-organic compounds are more environmentally friendly than already existing ones (e.g., our chlorine-free method for producing phosphorus-organic compounds). These technologies are less harmful to human health; so the amount of drugs needed by people will decrease. Furthermore, high-efficiency hemosorbents for hemodialysis, which are currently used in clinical practice, have been developed based on our divinyl sulfide (the product of the addition of hydrogen sulfide to acetylene). The first Russian pesticide, Vindidat, which was designed in our laboratory and is based on acetylene and sulfur compounds, was produced in the USSR on a pilot setup and is still used to protect residential and nonresidential areas and in the food industry.

- What pharmaceutical agents obtained via functionalization of heterocycles have reached production? Please tell us about them.

- Functionalization of heterocycles has for a long time been a common conventional approach in synthesizing various drugs. This refers to the drugs synthesized by my colleagues, academicians O.N. Chupakhin and V.N. Charushin, as well as to the drugs synthesized in our institute. For example, the previously mentioned drug Acizolum is a naturally abundant heterocycle functionalized by a vinyl group via direct interaction with acetylene. The new anti-tuberculosis drug perchlozone jointly designed by the researchers of our institute, St. Petersburg Research Institute of Phthisiopulmonology, and the company Farmasintez is also a very common heterocycle (pyridine) with thiosemicarbazide moiety introduced via a double bond. By the way, pyridines can be easily obtained via single-stage synthesis from acetylene and the simplest nitrogen-containing compounds (ammonia, amines, hydroxylamine, etc.). We have designed a convenient and simple method for the synthesis of pyridines from hydroxylamine, acetylene, and ketones. The reaction takes place in an aqueous alkaline medium; catalysis with heavy or noble metals, which is typical of this type of synthesis, is not required in this case.

A new group of functionalized pyrroles containing aromatic and heteroaromatic substituents with various functional groups that can be easily obtained using our reaction turned out to be another promising class of anti-tuberculosis agents. It has drawn the attention of many synthetic chemists working in pharmaceutical companies. Italian researchers have already found several leading agents. Some of them will apparently be sold in pharmacies soon. We are also working jointly with the pharmaceutical company Farmasintez in this direction.

One of the most marketable drugs, Atorvastatin (also known as Liprimar), is based on pyrroles with several benzene substituents and functional groups, which can also be obtained from ketones and acetylene through ketoximes, which allows one to eliminate several complex and labor-intensive stages in the conventional synthesis procedures. Atorvastatin is a widely known hypolipidemic agent; i.e., a drug that reduces the level of cholesterol in the blood.

Doctor of Chemical Sciences L.N. Sobenina and I have recently discovered a new simple method for introducing various functional groups to pyrroles and indoles using acetylenic ketones, acids, or their esters. A functionalized acetylene substituent is introduced into the pyrrole or indole ring when the reactants interact on active surfaces (e.g., upon grinding on aluminum oxide at room temperature in air). These reactions (known as cross coupling reactions) are typically carried out in the pres- ence of palladium catalysts and a number of additional reactants in an inert atmosphere upon heating. As I have already mentioned, the Nobel Prize was awarded in 2010 to R. Heck, E. Negishi, and A. Suzuki for the development of palladiumcatalyzed cross coupling reactions between acetylenes and halogen arenes. It is an interesting fact that another Nobel Prize was awarded in 2007 to G. Ertl for reactions on active surfaces.

A large number of modern drugs belong to the class of functionalized imidazoles. Therefore, the targeted introduction of the desired functional groups into the imidazole ring always remains a topical task for synthetic chemists and pharmaceutists. Acetylene chemistry helped us again. An original, general and very efficient strategy for functionalization of imidazoles via zwitterionic intermediates, adducts of imidazoles with cyanacetylenes, has been in systematic development in our laboratory for several years. This strategy allows one to simplify the synthesis of certain known drugs or their precursors and to synthesize new promising pharmaceutical agents.

- For which discovery did you receive the A.M. Butlerov Award?

- My pupil, Doctor of Chemical Sciences A.G. Mal'kina, and I received this award for a series of studies devoted to the synthesis of functionalized iminodihydrofuranes, which are key structural units and analogs of ascorbic and penicillic acids, natural cardiac glycosides, and certain synthetic agents exhibiting anticancer, antiulcerogenic, antiallergic, and anti-HIV activity. Iminodihydrofurane derivatives find application as nonsteroidal anti-inflammatory agents and analgesics.

We have designed simple and original methods for the synthesis of these compounds on the basis of cyanacetylene alcohols, which in turn have become widely available due to our systematic research. They are 
used in fine organic synthesis based on acetylene as convenient universal reactants. It is worth mentioning that Academician A.E. Favorsky, to whose school we belong, was the pupil closest to A.M. Butlerov. It was Butlerov who charged his PhD student Favorsky with developing the research area of acetylene. Thus, it is more correct to say that acetylene chemistry is the development of traditions, approaches, and methods of the traditional Russian Butlerov and Favorskys school.

- Boris Aleksandrovich, I would like to ask you about international collaboration. Are there any joint drug production projects?

- We have collaborated with a number of foreign universities and companies in the U.S., England, Germany, France, Spain, the Netherlands, Canada, Portugal, China, South Korea, and Taiwan. However, the collaboration is mainly oriented towards creating materials for modern technologies, including nanostructured materials, such as organic semiconductors, chemical current sources, highly sensitive sensors, electrochromic materials for thin-film flexible displays, field transistors, etc. International pharmaceutical companies prefer to negotiate on purchasing our patents or licenses for drugs (joint projects cannot be carried out because of the inertia of our bureaucracy). Until recently, an academic institute had no right to sell its final developments. Some points are becoming clearer today; however, a lot of obscure points still remain.

We are participating in an international project of the European Economic Community aimed at designing a new zinc-polymer accumulator based on ionic liquids. Research teams from universities and companies in England, France, Spain, Canada, Portugal, and the Netherlands also participate in the project. The accumulator is intended to be used for electric vehicles; i.e., to make the environment and mankind healthier. This project is not directly associated with drug synthesis; however, it is directed towards decreasing drug consumption. I think that its significance is no less than that of curing people who are suffocating in noxious exhaust gases emitted by cars, airplanes, motor ships, and diesel locomotives.

Very few people have given a thought to the fact that the combustion of 1 ton of carbon requires an additional 3 tons of oxygen, which is not replaced in time because of the cutting-down of trees by the timber industry. We are turning our planet into an artificial gas chamber; it is especially true for megalopolises.

- What works carried out by your pupils would you like to mention?

- I am very lucky to have many pupils who are really gifted people. Among those, there are 26 professors and doctors of sciences and more than 70 candidates of sciences. I am especially happy to be surrounded by young and talented pupils. One of them is Elena Yur'evna Schmidt. We recently discovered a new fundamental reaction of addition of ketones to acetylenes in the presence of superbases (there is a real chance that this reaction will be named after her in future). My other pupil is Andrei Viktorovich Ivanov, a young doctor of sciences who defended his doctoral thesis a year ago at only 30 (he apparently is the youngest doctor of sciences in the Siberian Branch of the RAS). He is one of my science deputies, he deals with innovations and youth policy. Olesya Aleksandrovna Shemyakina is one of my young pupils who has also defended her doctoral thesis this year (she is 32). Aleksandr Viktorovich Artem'ev, one of my youngest pupils, is now carrying out a breakthrough study devoted to the synthesis of organic compounds containing seleniumand phosphorus with consultations by Professor N.K. Gusarova. We hope that he will become Doctor of Science at the age of 27-28. So there are enough people who will develop our chemistry in future.

- Boris Aleksandrovich, how do you manage to combine research and administrative duties?

- It is pretty simple: when you have reliable and experienced deputies who have been tested through hard times, when you have highly qualified assistants: advisors, secretaries and research secretaries, who catch everything at once, who are proficient in several languages, who are on familiar terms with the computer, and who can type at the speed of sound and with high literacy (a rather rare phenomenon nowadays). All this is about my deputies and assistants. Thanks to them, I can visit the laboratory every day and grasp the details of almost each important experiment. I consider a day to be lost if I do not discuss current work with my colleagues next to a draft hood.

But speaking seriously, it is getting more and more difficult to combine research and administrative duties. Each day, the bureaucratic terror becomes worse. The number of documents (questionnaires, inquiries, reports, references, etc.) that should have been filled out yesterday or even two days earlier grows exponentially. In addition, new and even more convoluted documents are being constantly invented. In fact, this situation is rather sad. It makes experts do purposeless work (the same as driving nails with a microscope). A bureaucratic apparatus (even as huge one as ours) is incapable of processing this amount of documents. If this AllRussia paper deluge is not stopped, all our science will sink pretty soon.

But I would like to end our conversation on an optimistic note. I am sure that the paper deluge will be stopped because even the Flood ended in due time.

Interview by Maria Morozova 